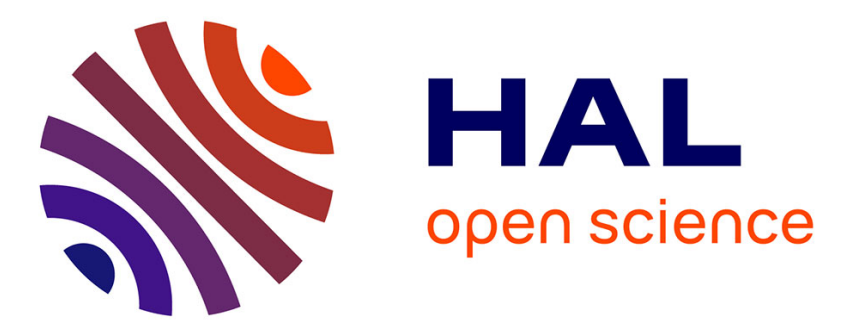

\title{
Multi-criteria Performance Analysis of Faster Than Nyquist Signaling
}

\author{
T. Petitpied, S. Traverso, Pascal Chevalier, R. Tajan, G. Ferre
}

\section{To cite this version:}

T. Petitpied, S. Traverso, Pascal Chevalier, R. Tajan, G. Ferre. Multi-criteria Performance Analysis of Faster Than Nyquist Signaling. 2018 25th International Conference on Telecommunications (ICT), Jun 2018, St. Malo, France. pp.42-46, 10.1109/ICT.2018.8464873 hal-02461662

\section{HAL Id: hal-02461662 \\ https://hal.science/hal-02461662}

Submitted on 30 Jan 2020

HAL is a multi-disciplinary open access archive for the deposit and dissemination of scientific research documents, whether they are published or not. The documents may come from teaching and research institutions in France or abroad, or from public or private research centers.
L'archive ouverte pluridisciplinaire HAL, est destinée au dépôt et à la diffusion de documents scientifiques de niveau recherche, publiés ou non, émanant des établissements d'enseignement et de recherche français ou étrangers, des laboratoires publics ou privés. 


\title{
Multi-criteria Performance Analysis of Faster Than Nyquist Signaling
}

\author{
T. Petitpied ${ }^{* \dagger}$, S. Traverso*, P. Chevalier*‡, R. Tajan ${ }^{\dagger}$, G. Ferré \\ * Thales Communications \& Security \\ 4 avenue des Louvresses, 92230 Gennevilliers (FRANCE) \\ $\dagger$ Univ. Bordeaux, Bordeaux INP, CNRS, IMS, UMR 5218, F-33405 Talence (FRANCE) \\ $\ddagger$ CEDRIC laboratory - CONSERVATOIRE NATIONAL DES ARTS ET MÉTIERS \\ 292 rue Saint-Martin, 75141 Paris Cedex 3 (FRANCE)
}

Email: forename.name@ $\left\{{ }^{*}\right.$ thalesgroup.com, ${ }^{\dagger}$ ims-bordeaux.fr, ${ }^{\ddagger}$ cnam.fr $\}$

\begin{abstract}
In this paper we propose a multi-criteria comparison of faster than Nyquist (FTN) against Nyquist signaling. To conduct this study we consider uncoded links and maximum likelihood receivers, we fix both the spectral efficiency (SE) and the bandwidth of the links, and we compare the quality of the latter for a given received signal to noise ratio (SNR) taking into account the peak-to-average power ratio (PAPR). Indeed, this metric directly affects the power amplifier (PA) efficiency and depends on the considered modulation and shaping filter. In addition, as already mentioned in the literature, the PAPR varies significantly with the compression factor of FTN signaling. Based on this, we built a penalized SNR expression in order to consider the PAPR effect and offer a clear representation to fairly compare different FTN and Nyquist signaling. Through this multi-criteria approach, required for practical applications, we show that the best waveforms are FTN. As an example, FTN links may provide a gain up to $2.5 \mathrm{~dB}$ for $2.5 \mathrm{bits} / \mathrm{s} / \mathrm{Hz}$ for a bit error rate (BER) of $10^{-4}$ compared to the best Nyquist link.
\end{abstract}

\section{INTRODUCTION}

Facing the emerge of communication technologies, it is necessary to develop new infrastructures able to hold a growing number of data and to improve transmitted information rates. For meeting the users needs, the fifth generation of mobile communications (5G) has to densify the cellular networks while reducing the energy consumption and optimizing the spectrum resources. Thus, those conflicting problems need to be solved maximizing the $\mathrm{SE}$, i.e. the information rate transmitted in a given bandwidth.

A solution to address this complex problem is to operate jointly at the transmitter and the receiver stages, optimizing existing waveforms. At the transmitter side, one issue is to limit the PAPR of the chosen waveform, to minimize the price to pay to prevent non linearities of the PA. At the receiver side, the problem is to optimize the compromise between performance and complexity.

Classically, the uncoded Nyquist links shape the information stream by a square-root Nyquist filter and use a matched filter at reception in order to maximize the SNR on the current symbol before decision. For raised-cosine Nyquist links, the SE depends on two parameters corresponding to the modulation order $k$, and to the rolloff parameter $\alpha$. Increasing the $\mathrm{SE}$ requires to increase $k$ or to decrease $\alpha$. However increasing $k$ requires to increase the SNR, and then the transmitted power, whereas decreasing $\alpha$ generally induces a PAPR increase. A trade-off has then to be found to optimize the global performance of the link.

In order to increase the SE of single-carrier communications, new waveforms called FTN signaling, alternative to Nyquist signaling, have been proposed by Mazo [1]. For a FTN link, the symbol period is compressed with respect to that of Nyquist signaling. This allows us to increase the SE at the expense of inter-symbol interference (ISI) at reception. FTN signaling then offers a third parameter to optimize the SE. The issue of FTN lies in determining whether the SE gain has more benefit than the potential loss coming from the ISI considering a constant transmitted power.

Mazo shows that weakly compressing the signal incurs no error rate increase at high SNR for a binary phase-shift keying (BPSK) communication filtered by a cardinal sine if it is processed by a maximum likelihood (ML) receiver. The lowest compression factor is called the Mazo bound and corresponds to a compression factor of 0.802 , which leads to a $25 \%$ bit rate increase. The Mazo bound has been extended in [2], by computer simulations, to commonly used low order constellations and other Nyquist filters, but remains valid only when considering nearly noise-free communications. More recently, FTN signaling has known a regain of attention in [3], [4], [5] and references therein. The PAPR behavior of FTN signaling, which has been studied in [6], [7] and [8], is much more different than those obtained with classical Nyquist links. Unfortunately, none of the previous analysis compares FTN and Nyquist signaling through a multi-criteria approach, which may limit the operational use of FTN signaling.

In this context, to overcome the state of art limitations, the purpose of the paper is to compare FTN and Nyquist signaling through a multi-criteria approach, mandatory to consider a potential operational use of FTN signaling. Due to a lack of space, we limit the analysis to uncoded links using a ML receiver. Under these assumptions, we fix the SE and the bandwidth of the links and we compare, for several values of the SNR, the BER at the output of the receiver for FTN and 
Nyquist links. To take into account the PAPR criterion in the analysis, we introduce a penalized SNR and we evaluate the output BER as a function of this penalized SNR, which seems to be unprecedented. Through this multi-criteria approach, we show that FTN signaling seem to be better than Nyquist ones.

The paper is organized as follows. Section II introduces the system model and the problem formulation. The criteria used in the analysis are introduced in Section III, and Section IV presents the results of the multi-criteria analysis. Finally section $\mathrm{V}$ aims to draw some conclusions and to describe some perspectives.

\section{SYSTEM MODEL AND PROBLEM FORMULATION}

We consider an uncoded baseband communication system operating over an additive white gaussian noise (AWGN) channel. The information is supposed to be a sequence of $N$ independent and identically distributed symbols $\left(a_{n}\right)_{n \in[0, N-1]}$ drawn from a complex centered alphabet $\mathcal{M}$ composed by $M=2^{k}$ symbols. Each symbol is sequentially transmitted every $T_{s}$ seconds. Classically, for FTN signaling, $T_{s}=\tau T$ where $\tau \in(0,1]$ is the compression factor and $T$ is the so-called Nyquist symbol period. The symbol stream is then shaped by the filter $g(t)$ to build the baseband waveform:

$$
s(t)=\sqrt{\tau} \sum_{n=0}^{N-1} a_{n} g\left(t-n T_{s}\right),
$$

where the factor $\sqrt{\tau}$ allows working with constant average power regardless of the value of $\tau$. Based on the AWGN channel assumption, the received signal is expressed as:

$$
y(t)=s(t)+w(t),
$$

where $w(t)$ denotes the AWGN perturbation with power spectral density $N_{0}$. Note that this additive model is true for satellite links in particular, but the following study is conducted without loss of generality.

Assuming equiprobable symbol sequences, the optimal receiver corresponds to the ML receiver [9], whose structure is depicted at Fig. 1, jointly with the transmitter part. This receiver is composed of a matched filter $g^{*}(-t)$ where $(\cdot)^{*}$ denotes the complex conjugate, a sampling operation every $T_{s}$ and a decision box implementing the Viterbi algorithm, which outputs the detected symbols $\left(\hat{a}_{n}\right)$. We deduce from (1) and (2) the output of the matched filter, given by:

$$
r(t)=\sqrt{\tau} \sum_{n=0}^{N-1} a_{n} v\left(t-n T_{s}\right)+w^{\prime}(t),
$$

where $w^{\prime}(t)=g^{*}(-t) \star w(t)$ and $v(t)=g^{*}(-t) \star g(t)$ is a Nyquist filter for $\tau=1$, with $\star$ the convolution product operator. Denoting by $r[n]$ the sample $r\left(n T_{s}\right)$ and $v[n]=$ $v\left(n T_{s}\right)$, we obtain for $\tau=1$ :

$$
r[n]=v[0] a_{n}+w^{\prime}[n]
$$

and for $\tau<1$ :

$$
r[n]=\sqrt{\tau}\left(v[0] a_{n}+\sum_{\substack{m=0 \\ m \neq n}}^{N-1} a_{m} v[n-m]\right)+w^{\prime}[n]
$$

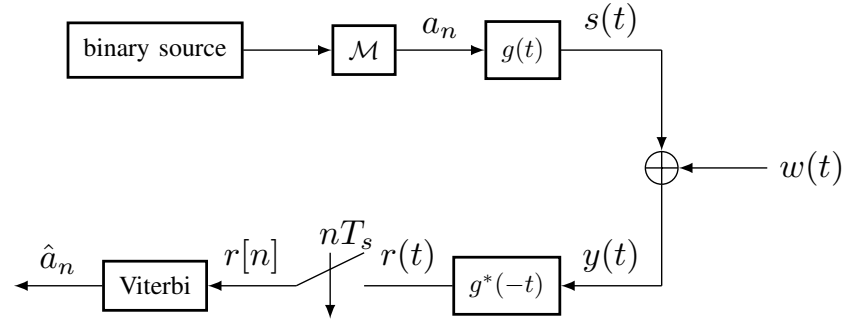

Fig. 1. System model

We choose in the following raised-cosine pulse shaping filters associated with the symbol duration $T$ for the filter $v(t)$. The link is then parametrized by four parameters corresponding to $k, \tau, \alpha \in[0 ; 1]$ the rolloff of $g(t)$, and $T$. For given values of the bandwidth $W$ defined in (6) and the SE $\rho$ defined in (7), the problem which is addressed in the paper consists in finding the best set of parameters $(k, \alpha, \tau)$ minimizing the BER for given values of the received SNR taking into account the PAPR.

\section{CRITERIA USED IN THE ANALYSIS}

The main criteria which characterize the links and which are used in our analysis correspond to the bandwidth $W$, the $\operatorname{SE} \rho$, the PAPR, the received SNR and the BER. We firstly present in this section the four first criteria. Then, assuming that the received SNR is the maximal SNR obtained with the PA used at transmission in linear regime, we propose a penalized SNR, denoted by $\mathrm{SNR}_{p}$, taking easily into account both the received SNR and the PAPR parameter.

\section{A. Bandwidth}

The bandwidth of Nyquist and FTN signaling depends on the Nyquist symbol period $T$ and the rolloff $\alpha$, and is defined by:

$$
W=\frac{1+\alpha}{T}(\mathrm{~Hz})
$$

\section{B. Spectral efficiency}

SE of Nyquist and FTN links is defined by:

$$
\rho=\frac{k}{(1+\alpha) \tau}(\text { bits/s/Hz) }
$$

\section{Received SNR}

The received SNR is the ratio between the useful power and the background noise power in the useful bandwidth at the output of the receive antenna. It is defined by:

$$
\mathrm{SNR}=\frac{P}{N_{0} W}
$$

where $P=\left\langle\mathbb{E}\left[|s(t)|^{2}\right]\right\rangle$, and $\langle\cdot\rangle$ corresponds to the temporal averaging over a time interval of length $T_{s}$. It is easy to verify that

$$
P=\frac{\sigma_{a}^{2}}{T} \int_{-\frac{W}{2}}^{\frac{W}{2}}|v(f)|^{2} d f,
$$

with $\sigma_{a}^{2}=\mathbb{E}\left[\left|a_{n}\right|^{2}\right]$ and $v(f)$ the Fourier transform of $v(t)$. 


\section{Peak-to-average power ratio}

The PAPR is defined as the ratio between the maximum instantaneous power and the mean power of the continuous signal to be transmitted. This factor depends on the considered modulation, the shaping filter and the FTN link compression factor $\tau$, and is straightforwardly given by:

$$
P A P R=\frac{\tau}{P} \max _{\left(a_{n}\right), t}\left|\sum_{n} a_{n} g\left(t-n T_{s}\right)\right|^{2}
$$

This ratio allows the communication designer to dimension the PA at the transmitter side to obtain the required received SNR without any saturation. Indeed, if we consider a linear response function of the PA, a backoff equivalent to the PAPR needs to be introduced. This allows to be able to send the most important peak power at the PA saturation level. For a given PA, this security interval directly affects the communication range because the transmitted mean power is linearly penalized. Moreover, for a given range, it requires to upgrade the PA and the cost of the transmitter.

However, considering the PAPR value to determine the PA cost appears to be overdimensioned since this value is rarely reached for single-carrier links. For this reason we focus on the instantaneous-to-average power ratio (IAPR) in our study, defined as (see [10]):

$$
p(t)=\frac{|s(t)|^{2}}{P}
$$

and more specifically to its time averaged complementary cumulative distribution function (CCDF) defined in (12), which allows us to access the time ratio the signal $p(t)$ is greater than a given threshold $\gamma^{1}$ [7].

$$
\operatorname{CCDF}(\gamma)=\frac{1}{T_{s}} \int_{0}^{T_{s}} \mathbb{P}(p(t)>\gamma) d t
$$

Fig. 2 shows for two values of the SE, the CCDF of IAPR for different $(k, \alpha, \tau)$ combinations. Indeed, the IAPR of some FTN solutions may be better than that of Nyquist links, depending on the given SE. Note that this result has already been observed in [6]. More precisely, Fig. 2 shows that a FTN link may bring a gain in IAPR with respect to Nyquist links greater than $2 \mathrm{~dB}$ for $\rho=2 \mathrm{bits} / \mathrm{s} / \mathrm{Hz}$ and greater than $1 \mathrm{~dB}$ for $\rho=2.5 \mathrm{bits} / \mathrm{s} / \mathrm{Hz}$, when considering a probability of $10^{-5}$ that the IAPR goes beyond $\gamma$. We conclude that around an extended Mazo bound for the parameter $\tau$, some FTN links may offer best IAPR than Nyquist links.

\section{E. Penalized received SNR}

In this subsection, for a given link, we propose to build a scalar criterion called penalized SNR, taking jointly into account the received SNR and the IAPR. This new criterion allows us to easily insert the IAPR parameter in the multicriteria comparison between FTN and Nyquist links.

\footnotetext{
${ }^{1}$ Note that the PAPR is then closely related to the IAPR since it corresponds to its maximum observed for any time and any sequence.
}

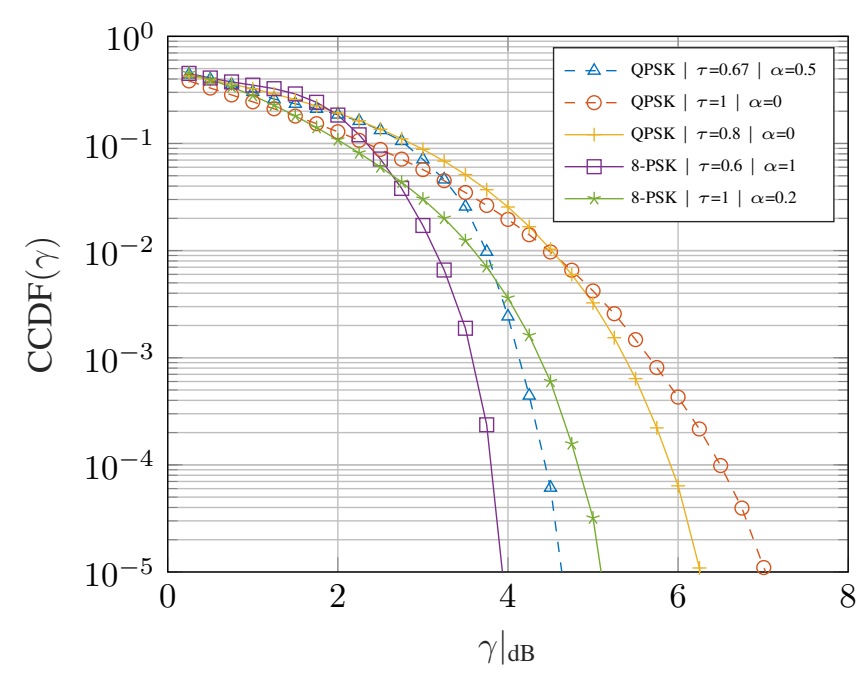

Fig. 2. IAPR CCDF for FTN and Nyquist links reaching $\rho=2$ bits $/ \mathrm{s} / \mathrm{Hz}$ (dashed) or $\rho=2.5 \mathrm{bits} / \mathrm{s} / \mathrm{Hz}$ (solid)

To this aim, we introduce, for a given link and a given probability $\theta$, a threshold IAPR, IAPR ${ }^{\theta}$, defined by (13) and allowing to consider only one IAPR value in the budget link.

$$
\operatorname{IAPR}^{\theta}=\min \{\gamma: \operatorname{CCDF}(\gamma) \leq \theta\}
$$

Moreover, we assume that, for a given link, the received SNR is the maximal SNR obtained with the PA used at the transmitter side in linear regime. Under this assumption, we deduce that the received SNR, defined in (8), is such that:

$$
P=\frac{P_{\text {sat }}}{\mathrm{IAPR}^{\theta}}
$$

where $P_{\text {sat }}$ is the output saturation power of the PA used at transmission. The $\mathrm{IAPR}_{\theta}$ then corresponds to the required input backoff ensuring a predefined saturation probability $\theta$. For a given PA (i.e. a given value of $P_{\text {sat }}$ ), the IAPR decreases $P$ and then the received SNR. Conversely, for a given received SNR (i.e. a given value of $P$ ), $P_{\text {sat }}$ has to increase linearly with the IAPR. This means that more powerful and more costly PA are proportionally required at transmission as the IAPR increases.

In this context, the penalized SNR, denoted by $\mathrm{SNR}_{p}$, for the considered link is defined by:

$$
\mathrm{SNR}_{p}=\mathrm{SNR} \cdot \mathrm{IAPR}^{\theta}=\frac{P_{\text {sat }}}{N_{0} W}
$$

This penalized SNR takes jointly into account both the received SNR and the IAPR. To insert it in the multi-criteria analysis it is sufficient to compute the BER at the output of the receiver as a function of $\mathrm{SNR}_{p}$ instead of $\mathrm{SNR}$.

\section{MUlTi-CRITERIA ANALYSIS}

We present in this section the multi-criteria comparison of FTN and Nyquist signaling. To this aim, we fix both the SE and the bandwidth of the links, and we compare the signaling performance through the illustration of the output BER as a function of the SNR. In the first subsection, the horizontal axis used is the received SNR whereas in the second subsection we 


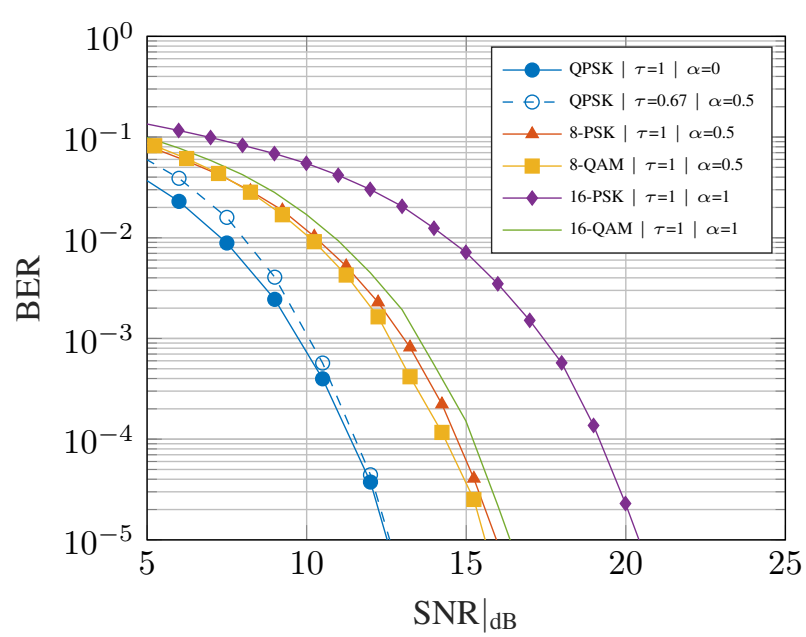

Fig. 3. BER performance comparison against $\left.\mathrm{SNR}\right|_{\mathrm{dB}}$ for FTN (dashed) and Nyquist (solid) signaling reaching $\rho=2 \mathrm{bits} / \mathrm{s} / \mathrm{Hz}$

consider the penalized SNR introduced in Subsection III-E. For the analysis, $g(t)$ is a root raised-cosine filter truncated at $\pm 3 T$.

\section{A. Comparative performance analysis without taking into account the IAPR}

For a given spectral occupation, the Nyquist link densifies the modulation to reach a potentially high SE. Conversely, the FTN link creates ISI by setting $\tau<1$ but increases the intersymbol distance by reducing the modulation order (decreasing $k$ ). The main issue in FTN is to solve the following problem: is the triplet $(k, \tau, \alpha)$ always optimal when $\tau=1$ ? In this paper we focus on answering this question considering the criterion detailed in Subsection III-E. Furthermore, (7) reveals that for a given $\mathrm{SE} \rho$, FTN a priori offers two alternatives to Nyquist signaling corresponding to:

- a modulation order $(k)$ decrease, compensated by low values of $\tau$ to reach a given value of $\rho$. This alternative is the only way a FTN solution can beat the best Nyquist one at a same SE and a given SNR,

- a rolloff increase, associated with low diminutions of $\tau$.

However, due to the presence of ISI at reception, we may wonder whether the error probability of the FTN links will be affected by low values of $\tau$. To answer to this question, we consider in this subsection several sets of parameters $(k, \tau, \alpha$, $T$ ) generating the same SE and bandwidth and we compare the output BER for several values of the received SNR.

We consider several values of $\rho$ and for each one, we focus on $k \in \llbracket 2 ; 2 \rho \rrbracket$ and $\alpha \in\{0,0.1, \ldots, 1\}$ and we adjust $\tau$ and $T$ such that $\rho$ and $W$ remain constant. For each modulation defined by $k$ and PSK or QAM, we plot the best FTN or Nyquist solution. Under these assumptions, Fig. 3 and Fig. 4 show, for several sets $(k, \tau, \alpha, T)$, the variations of the output BER as a function of the received SNR for $\rho=2 \mathrm{bits} / \mathrm{s} / \mathrm{Hz}$ and $\rho=2.5 \mathrm{bits} / \mathrm{s} / \mathrm{Hz}$ respectively.

Fig. 4 shows that for $\rho=2.5 \mathrm{bits} / \mathrm{s} / \mathrm{Hz}$, the best solution is a QPSK FTN with $(\tau, \alpha)=(0.8,0)$, allowing to obtain a 2.8 $\mathrm{dB}$ gain in the budget link with respect to the best Nyquist

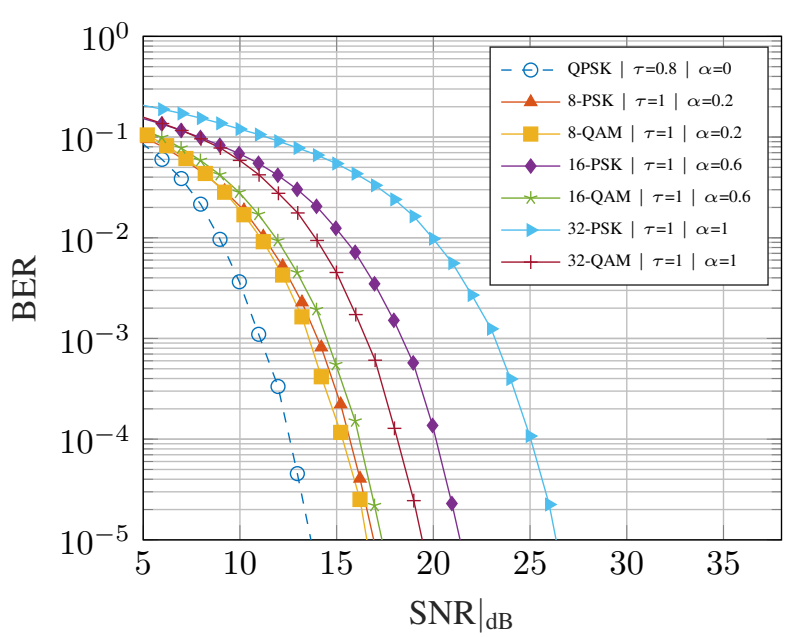

Fig. 4. BER performance comparison against $\left.\mathrm{SNR}\right|_{\mathrm{dB}}$ for FTN (dashed) and Nyquist (solid) signaling reaching $\rho=2.5 \mathrm{bits} / \mathrm{s} / \mathrm{Hz}$

option, a 8-QAM with rolloff $\alpha=0.2$. Note that $k$ cannot be an integer smaller than $\rho$ for a Nyquist alternative.

However, Fig. 3 shows that the conclusion changes for $\rho=$ 2 bits/s/Hz, which mitigates the results: the best link is a QPSK Nyquist solution with $\alpha=0$. Indeed, for a given SNR, a FTN solution cannot compete with an ISI free solution using a same modulation.

From the previous analysis, we may then be doubtful with respect to the interest of FTN links but the previous analysis ignores an important practical criterion which is the IAPR of the link, analyzed in the following subsection.

\section{B. Comparative performance analysis with the IAPR}

In this subsection, the IAPR criterion is inserted in the analysis through the penalized SNR defined in (III-E). Under the same assumptions of previous subsection, Fig. 5 and Fig. 6 show the same variations as in Fig. 3 and Fig. 4 respectively, for $\rho=2 \mathrm{bits} / \mathrm{s} / \mathrm{Hz}$ and $\rho=2.5 \mathrm{bits} / \mathrm{s} / \mathrm{Hz}$ respectively, but as a function of $\mathrm{SNR}_{p}$ with $\theta=10^{-5}$ instead of SNR.

In Fig. 5, the IAPR gives the advantage to a weakly compressed QPSK solution such that $(\tau, \alpha)=(0.71,0.4)$ which generates a gain of $1.3 \mathrm{~dB}$ with respect to the best Nyquist solution: a 8-PSK with $\alpha=0.5$. Moreover, according to our results, the best FTN solution has a compression factor equivalent to Mazo bound for such a modulation and rolloff. These results are confirmed on Fig. 6 for $\rho=2.5$ bits $/ \mathrm{s} / \mathrm{Hz}$ for which the best link is a QPSK FTN alternative such that $(\tau, \alpha)=(0.8,0)$. If looking at same modulation orders as the best Nyquist link $(k=3)$, the best solution is an other FTN solution such that $(\tau, \alpha)=(0.6,1)$. The first FTN link offers a gain of $2.5 \mathrm{~dB}$ over the best Nyquist alternative, a 8-PSK with $\alpha=0.2$ whereas the gain offered by the second is $1 \mathrm{~dB}$.

These results show that if we can afford a ML receiver to overcome the ISI, a low compression factor can offer a low IAPR and an overall gain for uncoded communications. 


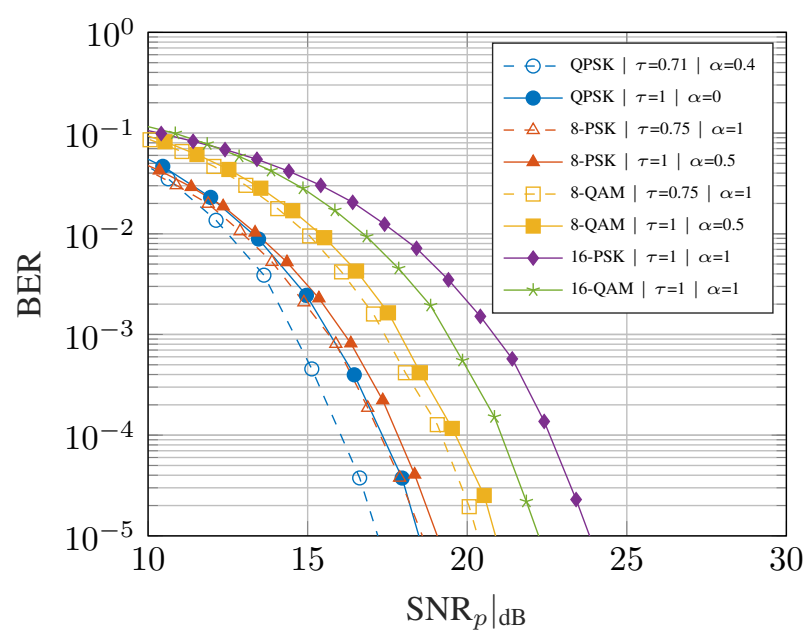

Fig. 5. BER performance comparison against $\left.\mathrm{SNR}_{p}\right|_{\mathrm{dB}}$ with $\theta=10^{-5}$ for FTN (dashed) and Nyquist (solid) signaling reaching $\rho=2 \mathrm{bits} / \mathrm{s} / \mathrm{Hz}$

TABLE I

GAIN USING FTN RATHER THAN NYQUIST AT BER $=10^{-4}$ AND $\theta=10^{-5}$

Spectral efficiency $\rho$ (bits/s/Hz)

\begin{tabular}{|c|c|c|c|c|}
\multicolumn{1}{c|}{} & 1.5 & 2 & 2.5 & 3 \\
\hline$(k, \tau, \alpha)$ & $(2,0.67,1)$ & $(2,0.67,0.5)$ & $(2,0.8,0)$ & $(2,0.67,0)$ \\
\hline $\mathrm{SNR}_{\text {gain }}$ & $0 \mathrm{~dB}$ & $-0.2 \mathrm{~dB}$ & $2.8 \mathrm{~dB}$ & $1.2 \mathrm{~dB}$ \\
\hline $\mathrm{SNR}_{p}$ gain & $0.4 \mathrm{~dB}$ & $1.3 \mathrm{~dB}$ & $2.5 \mathrm{~dB}$ & $1.9 \mathrm{~dB}$ \\
\hline
\end{tabular}

\section{General comparisons}

The results presented in previous subsections can be generalized to other values of the SE. Indeed, Table I indicates, for several values of the $\mathrm{SE}$, the gain in $\mathrm{SNR}$ and $\mathrm{SNR}_{p}$ obtained in using FTN instead of a Nyquist signaling. The parameters of the associated FTN solutions are also indicated. Results for a constrained transmit power can be obtained by fixing the SNR or penalized SNR and comparing the BER.

For $\rho \in\{1.5,2\}$, the Nyquist solutions are always better than the FTN ones if we do not take into account the IAPR. The optimal Nyquist link use, in this case, a QPSK modulation with a rolloff $\alpha$ adjusted to obtain the given $\rho$. However, if we take into account the IAPR, the best solutions become QPSK FTN alternatives which offer best IAPR performances.

For $\rho \in\{2.5,3\}$, FTN is always better than Nyquist signaling, for both the $\mathrm{SNR}$ and the $\mathrm{SNR}_{p}$ criteria, i.e. with and without taking into account the IAPR. In this case, FTN allows to decrease the modulation order with respect to Nyquist thanks to the decrease of $\tau$.

\section{CONCLUSION}

In this paper, uncoded FTN and Nyquist signaling have been compared through a multi-criteria approach, required for practical implementations using a ML receiver. This allows us to take into account the IAPR of the links, through a penalized received $\mathrm{SNR}$ noted $\mathrm{SNR}_{p}$, which is unprecedented up to our knowledge. Through this analysis including the IAPR, FTN

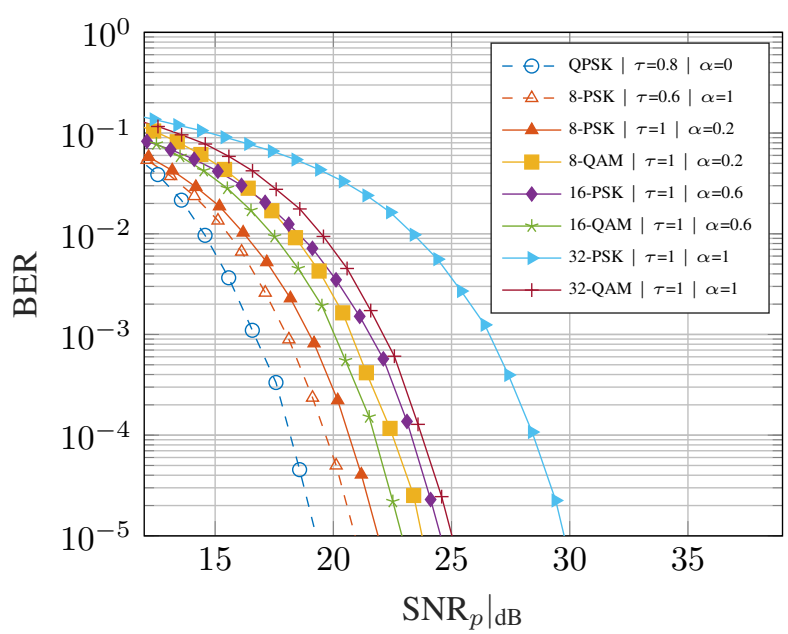

Fig. 6. BER performance comparison against $\left.\mathrm{SNR}_{p}\right|_{\mathrm{dB}}$ with $\theta=10^{-5}$ for FTN (dashed) and Nyquist (solid) signaling reaching $\rho=2.5 \mathrm{bits} / \mathrm{s} / \mathrm{Hz}$

solutions have been shown to be better than Nyquist ones for SE between 1.5 and 3 bits/s/Hz. Optimal FTN solutions have been shown to use a compression factor $\tau \in[0.6,0.8]$ and may generate $\mathrm{SNR}_{p}$ gains up to $2.5 \mathrm{~dB}$. Future works will consider higher SE and will take into account in the analysis both coding links and alternative receivers with a reduced complexity.

\section{REFERENCES}

[1] J. E. Mazo, "Faster-than-Nyquist signaling," The Bell System Technical Journal, vol. 54, no. 8, pp. 1451-1462, Oct 1975.

[2] A. D. Liveris and C. N. Georghiades, "Exploiting Faster-than-Nyquist signaling," IEEE Transactions on Communications, vol. 51, no. 9, pp. 1502-1511, Sept 2003.

[3] J. B. Anderson, F. Rusek, and V. Öwall, "Faster-than-nyquist signaling," Proceedings of the IEEE, vol. 101, no. 8, pp. 1817-1830, Aug 2013.

[4] A. Barbieri, D. Fertonani, and G. Colavolpe, "Time-frequency packing for linear modulations: spectral efficiency and practical detection schemes," IEEE Transactions on Communications, vol. 57, no. 10, pp. 2951-2959, October 2009.

[5] D. Dasalukunte, F. Rusek, and V. Öwall, "A 0.8 mm2 9.6 mw implementation of a multicarrier faster-than-nyquist signaling iterative decoder in 65nm cmos," in 2012 Proceedings of the ESSCIRC (ESSCIRC), Sept 2012, pp. 173-176.

[6] J. A. Lucciardi, N. Thomas, M. L. Boucheret, C. Poulliat, and G. Mesnager, "Trade-off between spectral efficiency increase and papr reduction when using ftn signaling: Impact of non linearities," in 2016 IEEE International Conference on Communications (ICC), May 2016, pp. 17.

[7] T. Delamotte, A. Knopp, and G. Bauch, "Faster-than-Nyquist signaling for satellite communications: A papr analysis," in SCC 2017; 11th International ITG Conference on Systems, Communications and Coding, Feb 2017, pp. 1-6.

[8] A. Liu, S. Peng, L. Song, X. Liang, K. Wang, and Q. Zhang, "Peakto-average power ratio of multicarrier faster-than-nyquist signals: Distribution, optimization and reduction," IEEE Access, vol. 6, pp. 11977 $11987,2018$.

[9] G. Ungerboeck, "Adaptive maximum-likelihood receiver for carriermodulated data-transmission systems," IEEE Transactions on Communications, vol. 22, no. 5, pp. 624-636, May 1974.

[10] C. Ciochina, F. Buda, and H. Sari, "An analysis of OFDM peak power reduction techniques for WiMAX systems," in 2006 IEEE International Conference on Communications, vol. 10, June 2006, pp. 4676-4681. 